\title{
Non extensive description of $p p$ collisions at high energies
}

\author{
M. Abdel-Aziz ${ }^{1,2}$, S. Abdel-Razik ${ }^{3}$ and M. Sirag ${ }^{3}$ \\ ${ }^{1}$ Basic Science Department ,Faculty of Computers and Information Sciences, Ain \\ Shams University, Khalifa El-Maamon St, Cairo-11566, Egypt \\ ${ }^{2}$ Scientific Computing Center ,Faculty of Computers and Information Sciences, Ain \\ Shams University, Khalifa El-Maamon St,Cairo-11566, Egypt \\ ${ }^{3}$ Physics Department, Faculty of Women, Ain Shams University, AsmaaFahmi, \\ Al Golf, Nasr City, Cairo-11566, Egypt
}

\begin{abstract}
Non extensive statistics provide an excellent application in the field of high energy collisions to study the thermal properties of the produced particles. We apply the scaled Tsallis equation to study the entropy parameter as a function of transverse momentum of the produced hadrons. We applied Tsallis-Pareto to study the transverse momentum distribution of produced hadrons $\left(\pi^{ \pm}, k^{ \pm}, p, \bar{p}\right)$ in pp collisions at center of mass energies $\sqrt{S_{N N}}=62.4,200,900 \mathrm{GeV}$ and $2.7 \mathrm{TeV}$. The Pareto-Tsallisdistribution, leads to an excellent description of data on transverse momentum distribution. The qparameters are almost independent from $\sqrt{s}$ for protons and kaons and distributed around $q=1.1$ and $\mathrm{q}$ is increasing for pions from 1.1 to almost 1.3 at top energy $\sqrt{s}=2.7 \mathrm{TeV}$. The effective temperature, $T$ increases with collisions energy we estimated $T \approx 0.3 \mathrm{GeV}$ for pp collisions at $2.7 \mathrm{TeV}$ LHC energy. Different hadrons have different effective temperature such that heavier hadrons have higher freezout temperature.
\end{abstract}

\section{Key words:}

Transverse momentum distribution, high energy collision, The Pareto-Tsallis distribution, Non extensive statistics, QGP.

\section{Introduction}

The transverse momenta of secondary identified particles produced in pp collisions is very important. Since QCD description of low $p_{t}$ particles is not available because ofnon perturbative effects. Phenomenological models has been proposed to solve thisproblem of description $p_{t}$ distribution in pp and nucleus-nucleus collisions. For exampleat low $p_{t}$ where the spectra is in the form of exponential decay hadron gas model withconventional Boltzmann Gibbs distribution are widely used to describe $p_{t}$ spectra andmultiplicity distribution. For high $p_{t}$ particles, the exponential BG distribution is notappropriate. Many authors proposed Tsallis distribution as an alternative to describe $p_{t}$ distribution of produced hadrons in $\mathrm{pp}$ collisions in terms of the temperature $T$ andthe nonextensive parameters $q$ which measures the degree of equitation of the fireball.

Corresponding author:Shimaa_m_elrazik@hotmail.com 
Tsallis distribution with thermodynamic consistent formulation is same as the formof QCD inspired Hagedorn distribution in terms of power $n$ related to $q$ [R. Hagedorn et al, 1983]. The high $p_{t}$ hadrons coming from initial hard scattering decide the value of $n$ and the low $p_{t}$ hadrons form the bulk of the spectra decideT. Moreover, to allow the nonextensiveparameter $q$ to change with hadron $p_{t}$. We use the same ansatz as in [[A] G. G. Barnafoldi et al, 2011]. The ansatzused in the current work is different form the one in [[B] G. G. Barnafoldi et al, 2011] as we will discuss in the restof this paper. The paper is organized as follows: Sec. 2 gives details about the model.In Sec.3 we present the fit to the $p_{t}$ spectra of differ hadrons .In Sec.4 we discuss the model parameters as a functions of hadron mass and energy for both positive andnegative hadrons. In last section our concluding remarks are given.

\section{Tsallis Distribution}

In this section will introduce a very brief discussion on tsallis distribution and discuss the need for a scaled tsallis distribution that allow for variation of the transverse momentum distribution of the secondary hadrons produced in $\mathrm{pp}$ at high energy. In high energy physics a thermodynamic consistent form of Tsallis statistics is needed to describe system very close to equilibrium [J. Cleymans, 2017].

Let's introduce the standard form of particlenumber $N$, the energy $E$ and the pressure $P$ is given by thefollowing expressions:

$$
\begin{aligned}
& N=g V \int \frac{d^{3} p}{(2 \pi)^{3}}\left[1+(q-1) \frac{E-\mu}{T}\right]^{-\frac{q}{q-1}}, \\
& E=g \int \frac{d^{3} p}{(2 \pi)^{3}} E\left[1+(q-1) \frac{E-\mu}{T}\right]^{-\frac{q}{q-1}}, \\
& P=g \int \frac{d^{3} p}{(2 \pi)^{3}} \frac{p^{2}}{3 E}\left[1+(q-1) \frac{E-\mu}{T}\right]^{-\frac{q}{q-1}}, \\
& \in+P=T s+\mu n
\end{aligned}
$$

Where $n, \in$, srefer to the densities of corresponding quantities. Standard Tsallis equation is shown in Eq.(1). Where $T$ and $\mu$ are the temperature andthe chemical potential, Vis the volume and $g$ is the degeneracy factor. This equation has $q$ independent $p_{t}$. The parameter $q$ is the entropy index that measures the deviationof the distribution from thermal one and is related to temperature fluctuations via

$$
\mathrm{q}-1=\frac{\left\langle\mathrm{T}^{2}\right\rangle-\langle\mathrm{T}\rangle^{2}}{\langle\mathrm{~T}\rangle^{2}}
$$


So $q$ can be a measure of the degree of thermalization in the created QGP medium that is produced in high energy nuclear collisions at RHIC and LHC. The particle number $N$ is related to the pressure and particle density $n$ by the thermodynamic formula

$$
N=\left.V \frac{\partial P}{\partial \mu}\right|_{T}
$$

It is worth to mention that, the above formulation does not include radial or elliptic flow. So the function distribution in Eq.(8) has no $\varphi$ dependence of even radial flow $\beta$ since these quantities are almost zero in case of $p p$ collisions in this case the temperature parameter $T$ can be interpreted as effective temperature.

In order to introduce the $p_{t}$ dependence of the $q$ we want to mention that very recent results produced by the recombination models [R. J. Fries et al,2005] reveals that the recombination ofconstituent quarks at intermediate $p_{t}$ is essential to describe the elliptic flow parameters $v_{2}, \pi /$ Pratio and nuclear modification factors $R_{A A}$. This investigation reveals that we can probe this variation as a function of particles transverse momentum distribution. To allow for such dependence we use the following ansatz [[A]G. G. Barnafoldi et al ,2011,[[B]G. G. Barnafoldi et al ,2011]:

$$
\alpha \rightarrow \alpha\left(p_{t}\right)=\frac{1}{1-q}-A \log \log \left(1+k \cdot p_{t}\right)
$$

So, now the standard Tsallis equations turn to be

$$
\frac{d^{2} N}{2 \pi d y p_{t} d p_{t}}=C m_{t}\left[1+\frac{m_{t}}{\alpha T}\right]^{-\alpha}
$$

Here $C=g V /(2 \pi)^{2}$ is the overall constant, with $g$ is the degeneracy and $\mathrm{V}$ is the volume. $m_{t}=\sqrt{m^{2}+p_{t}^{2}}$ is the transverse mass of the hadrons. In order to fit the experimental data and assess the quality of the fit. We use $\chi^{2}$ as follows

$$
\chi^{2}=\sum_{i=0}^{n_{o b s}} \frac{\left(O_{\text {exp }}\left(p_{t i}\right)-O_{\text {theory }}\left(p_{t i}\right)\right)^{2}}{\sigma_{i}^{2}}
$$

Where $O_{\text {exp }}\left(p_{t i}\right), O_{\text {theory }}\left(p_{t i}\right)$ stand for the measured value of $p_{t}$ distribution and the corresponding theoretical function evaluated at the same $p_{t}$ using Eq. (8) and $\sigma_{i}^{2}$ corresponds to combined statistical and systematic errors and $n_{o b s}$ is the number of data points.

\section{Results}

We apply Eq.(8) to fit the experimental data using $\chi^{2}$ fit. We use the standard ROOT class TMinuit to perform the $\chi^{2}$ fit. We start by applying the modified Tsallis distribution Eq.(8) to study transverse momentum distribution $\frac{d^{2} N}{2 \pi d y p_{t} d p_{t}}$ of particles produced in high 
energy experiments at relativistic heavy ion collider RHIC at energies 62.4 and $200 \mathrm{GeV}$ and at large hadron collider LHC at energies $900 \mathrm{GeV}$ and $2.7 \mathrm{TeV}$.

In the current work, We focus on the $p_{t}$ distribution of $\pi^{ \pm}, k^{ \pm}, p, \bar{p}$ produced in $p p$ collisions at RHIC and LHC. We use Eq. (8) to fit the $p_{t}$ distribution and get a systematic studies on how the model parameters and average transverse momentum $\left\langle p_{t}\right\rangle$ change with collisions energies and hadron mass.

First we study RHIC results of $p p$ collisions at center of mass energies $\sqrt{s}=62.4$ and $200 \mathrm{GeV}$ as measured by PHENIX [A. Adare et al,2011] at mid rapidity $y=0$.

In fig. 1 we use eq. (8) to fit the transverse momentum distribution of $\pi^{ \pm}, k^{ \pm}, p, \bar{p}$ produced in $p p$ collisions at $\sqrt{s}=62.4 \mathrm{GeV}$ as measured by PHENIX Eq.(8). It is clear that the experimental data are well reproduced within the measured transverse momentum $0.140 \leq p_{t} \leq 3$. In order to investigate the applicability of our model to higher energies used in RHIC, we apply Eq. (8) to the same particles as those in fig. 1 but in $p p$ collisions at different energies $\sqrt{s}=200$ as measured by PHENIX [A. Adare et al,2011]fig.2.

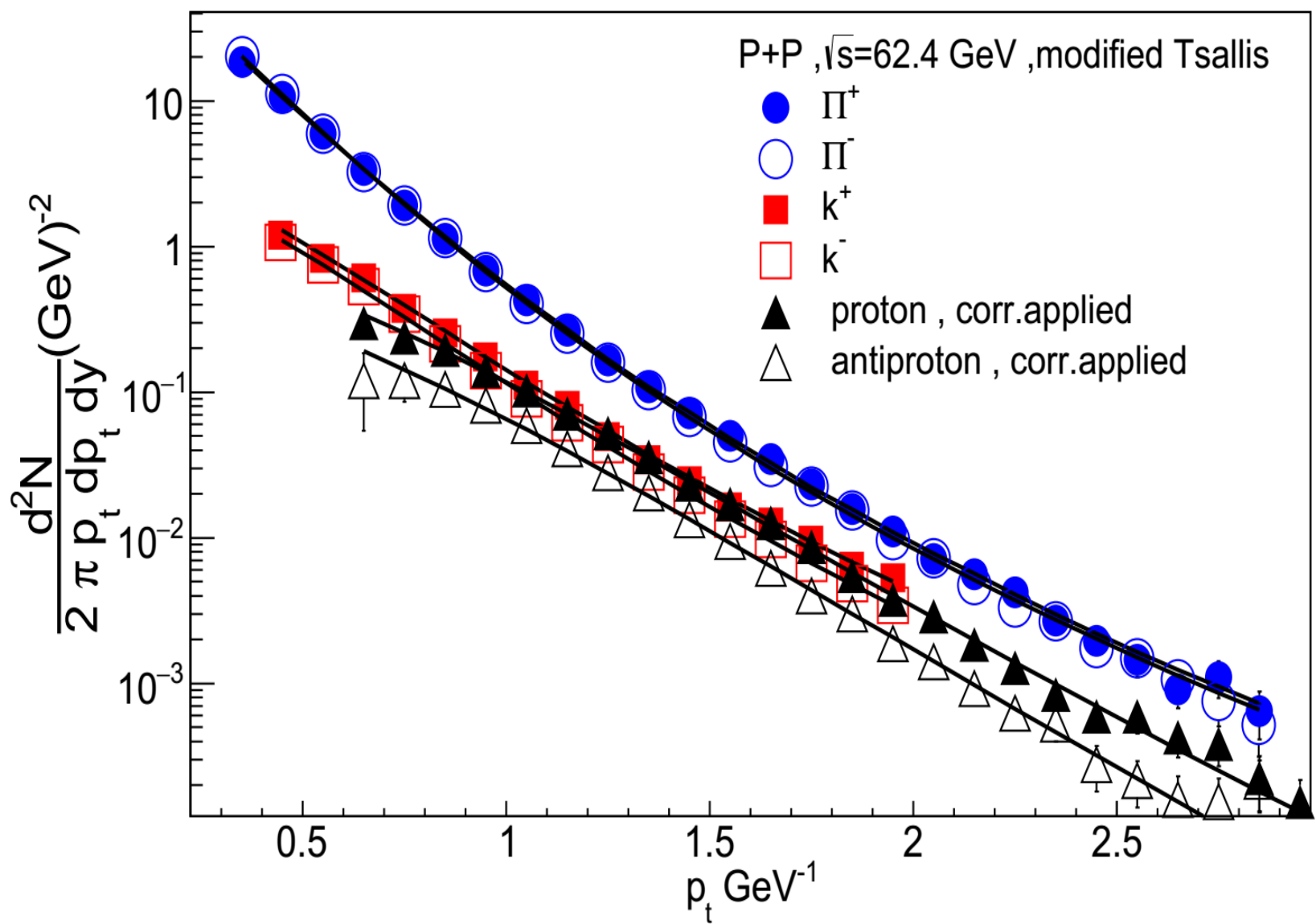

Figure 1: Transverse momentum distribution of $\pi^{+}, \pi^{-}, k^{+}, k^{-}$, proton and antiproton produced in $p p$ collisions as obtained by the PHENIX Collaboration [A. Adare et al,2011] at $\sqrt{s}=62.4 \mathrm{GeV}$ at midrapidity. Thesolid curves are the fits of the data of the modified Tsallis Eq. (8) at mid rapidity. 


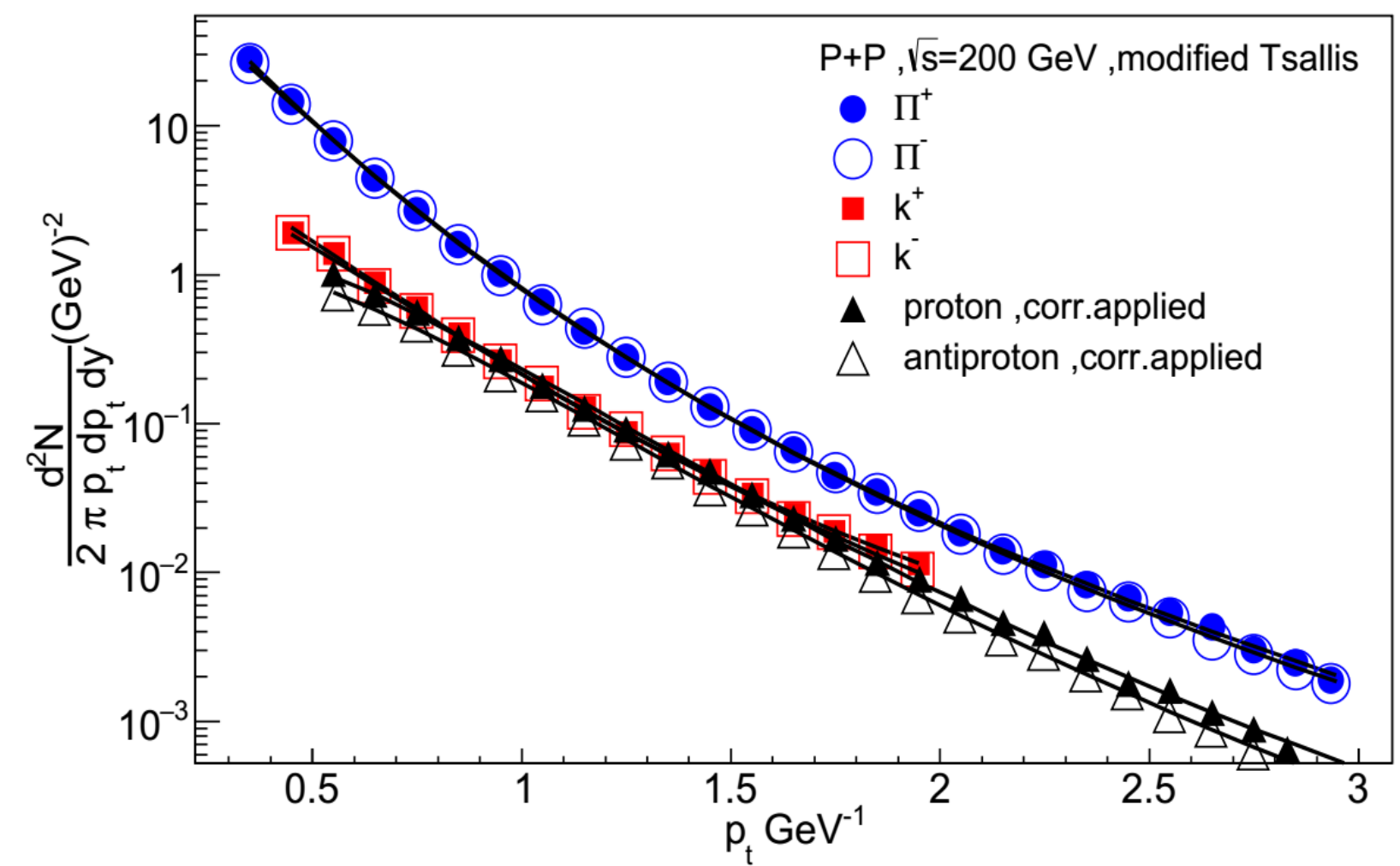

Figure 2: Transverse momentum distribution of $\pi^{+}, \pi^{-}, k^{+}, k^{-}$,proton and antiproton produced in pp collisions as obtained by the PHENIX Collaboration [A. Adare et al,2011] at $\sqrt{s}=200 \mathrm{GeV}$ at mid rapidity. The solid curves are the fits of the data of the modified Tsallis Eq.(8) at mid rapidity.

As shown in fig.(2) both pareto-tsallis model and the experimental data are inexcellent agreement within the given $p_{t}$ range $0.5 \mathrm{GeV}<p_{t}<3.0 \mathrm{GeV}$.

Both figures are clearly indicate that the transverse momentum, distribution of $\pi^{ \pm}, k^{ \pm}, p$, $\bar{p}$ produced in pp collisions at RHIC at two different energies are well produced.

To explore the applicability of the model to higher energies at LHC, we use the recent data measured by ALICE at LHC for pp collisions at $900 \mathrm{GeV}$. In Fig.3,we use Eq.(8) to fit the data of identified hadron produced in pp collisions at $900 \mathrm{GeV}$ in LHC as measured by [K. Aamodt et al, 2011]. We see good agreement between measured values and Eq.(8) for the measured range $0.1 \mathrm{GeV}<p_{t}<2.5 \mathrm{GeV}$. In fig.4, we show the results of applying Eq.(8) to fit the charged particles distribution $\pi^{+}+\pi^{-}, k^{+}+k^{-}, p+\bar{p}$. For $\pi^{+}+\pi^{-}$agreement between model and data is appealing up to $p_{t} \approx 18 \mathrm{GeV}$. While for pp and $k^{+}+k^{-}$the deviation starts to appear at about $p_{t} \approx 4 \mathrm{GeV}$. Recently, [G. Wilk and Z. Wlodarczyk,2017], [G. Wilk and Z. Wlodarczyk,2015], showed that the inclusion of oscillating factor in the nonextensive parameters can handle the oscillation founded in fitting the experimental data for hadrons produced during pp collisions. We will not go into the details of the nature of this oscillation because it outside the scope of the current work. 


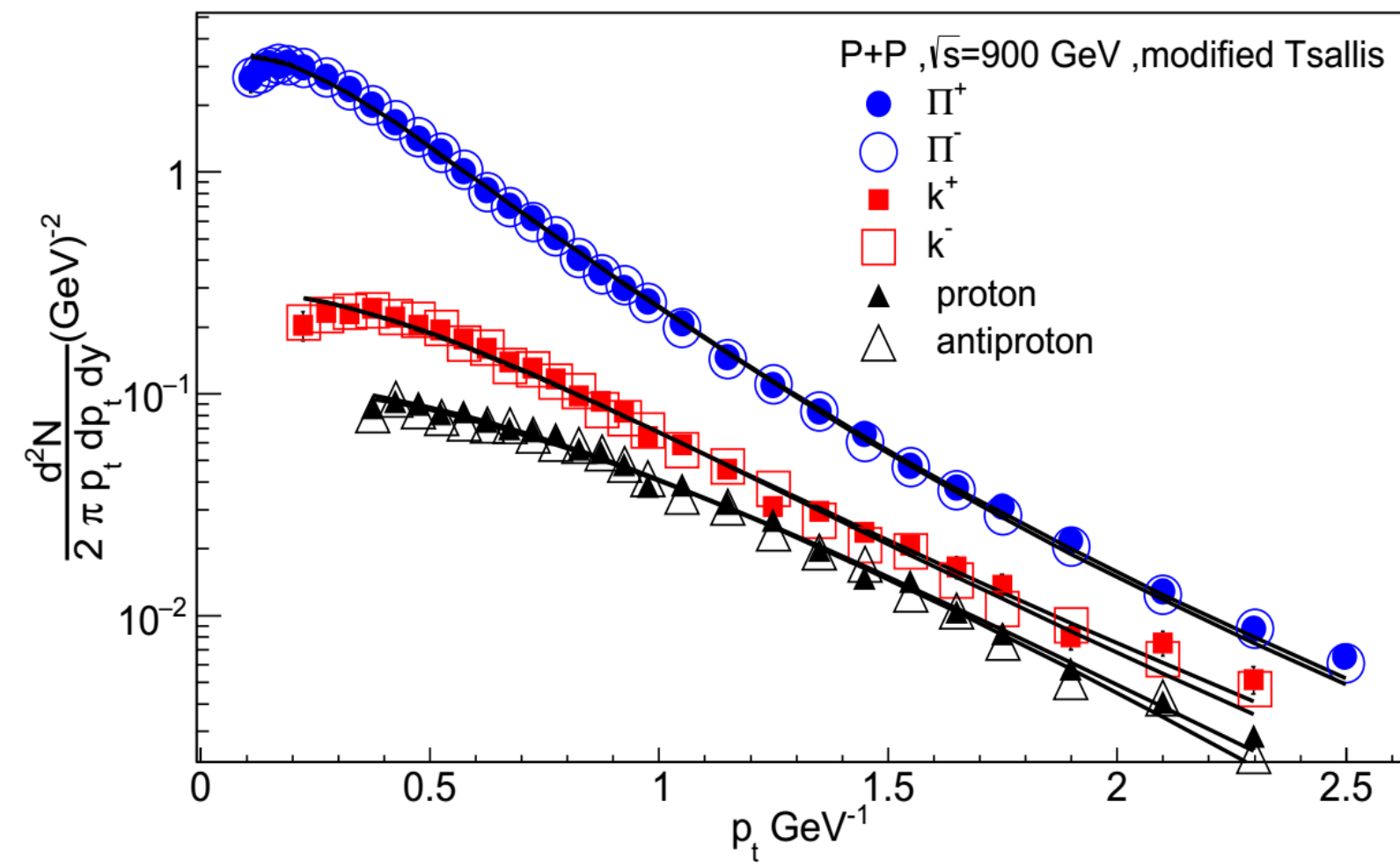

Figure 3: Transverse momentum distribution of of $\pi^{+}, \pi^{-}, k^{+}, k^{-}$, proton and antiproton produced in $p p$ collisions as obtained by ALLC Collaboration [K. Aamodt et al, 2011] at $\sqrt{s}=$ $900 \mathrm{GeV}$ at midrapidity. The solid curves are the fits of the data of the modified Tsallis Eq.(8) at mid rapidity.

\section{Model Parameters}

In previous section we presented the results of fitting the $p_{t}$ distribution of differentcharged hadrons. In this section will explore the model parameters and their significanceand how they vary as a function of collisions energy and hadron mass and also commentson the accuracy of our fit procedures. In tables $(1,2,3,4)$ we show the computed parameters in Eq.(8) with the corresponding $c h i^{2} / n d f$, where $n d f$ is the number ofdegrees of freedom, for fitting $p p$ data at $62.4,200,900 \mathrm{GeV}$ and $2.7 \mathrm{TeV}$. We noticefor every particles in those tables the corresponding $\chi^{2} / N D F$ is less than 1.0 that giveus confidence the model performs very well over the given collisions energy for everyhadrons in the tables. In Figs $(5,6)$ we show the extracted values of the nonextensiveparameter $q$ from as a function of collisions energy for both negative and positive hadronsrespectively. The nonextensive parameters q does not show energy dependance withinthe error bars but for both $\pi^{ \pm}$it is clearly $q$ increases with the collisions energy from $q=1.1$ at 62.4 $\mathrm{GeV}$ to $q=1.3$ at $900 \mathrm{GeV}$. 


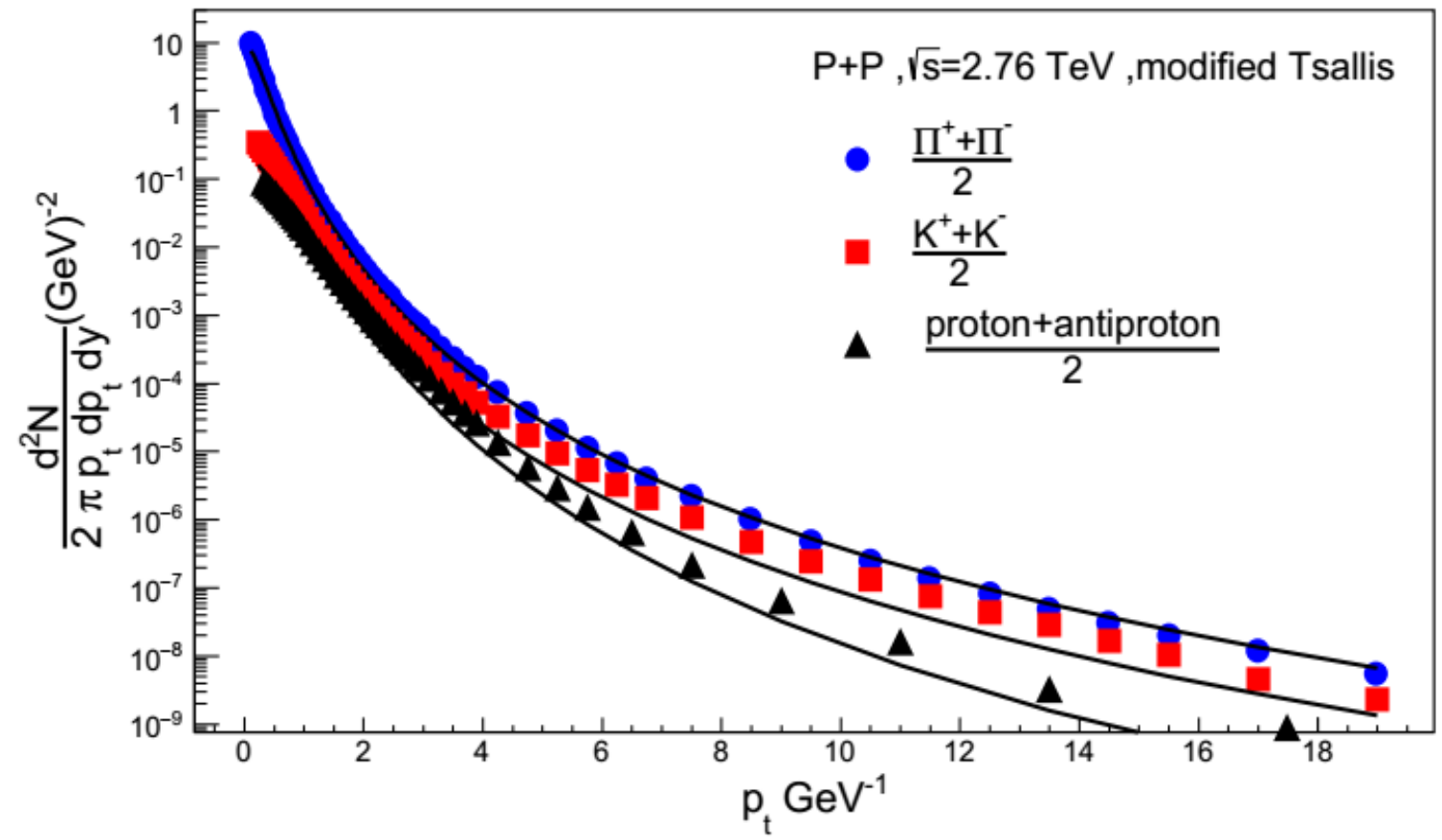

Figure 4: Transverse momentum distribution of $\pi^{+}, \pi^{-}, k^{+}, k^{-}$, proton and antiproton produced inpp collisions as obtained by ALLC Collaboration at $\sqrt{s}=2.7 \mathrm{TeV}$ at midrapidity. The solid curves arethe fits of the data of the modified Tsallis Eq.(8) at mid rapidity.

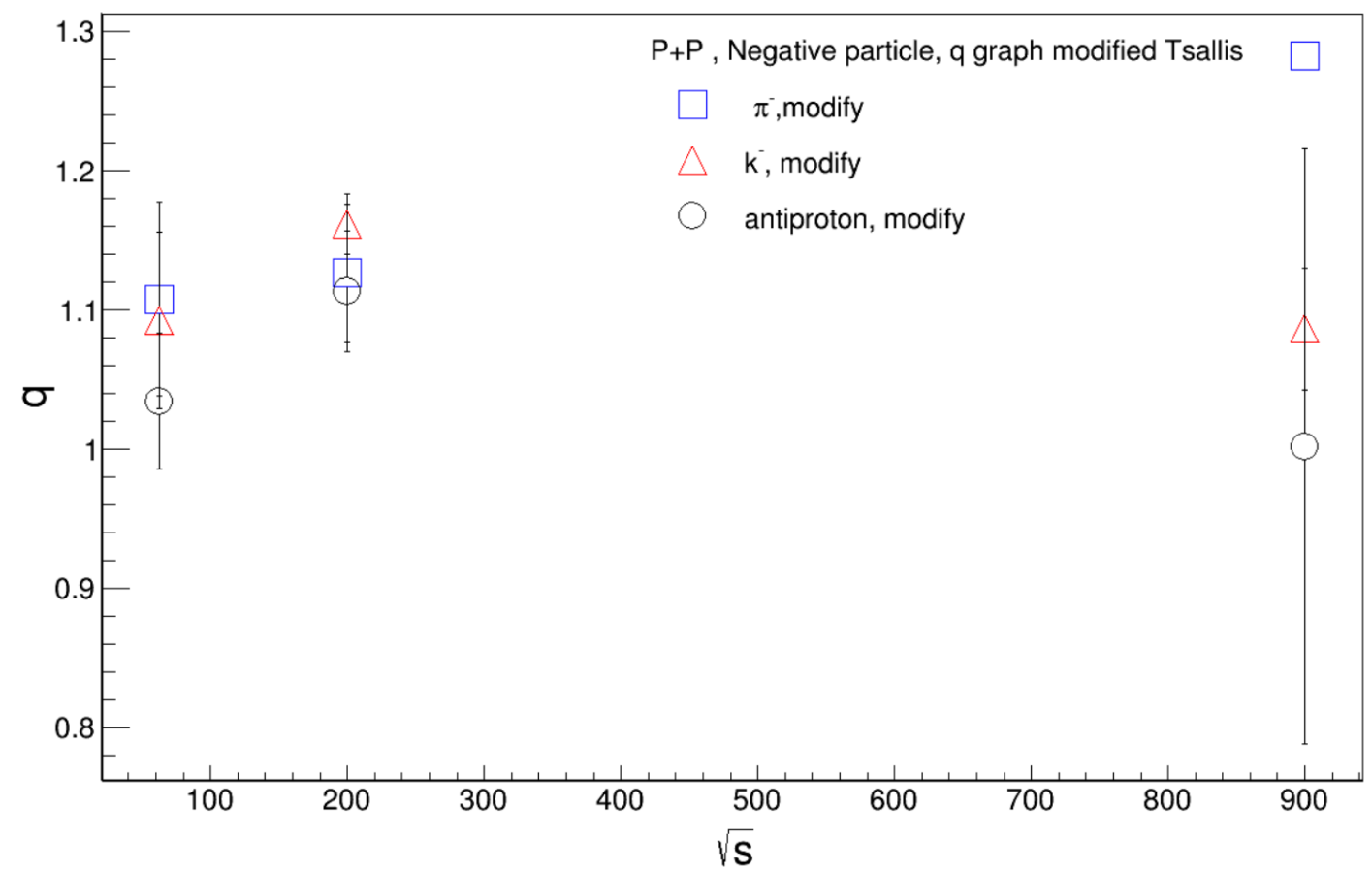

Figure 5: The nonextensive parameter $q$ for-vehadrons versus $\sqrt{s}$ 


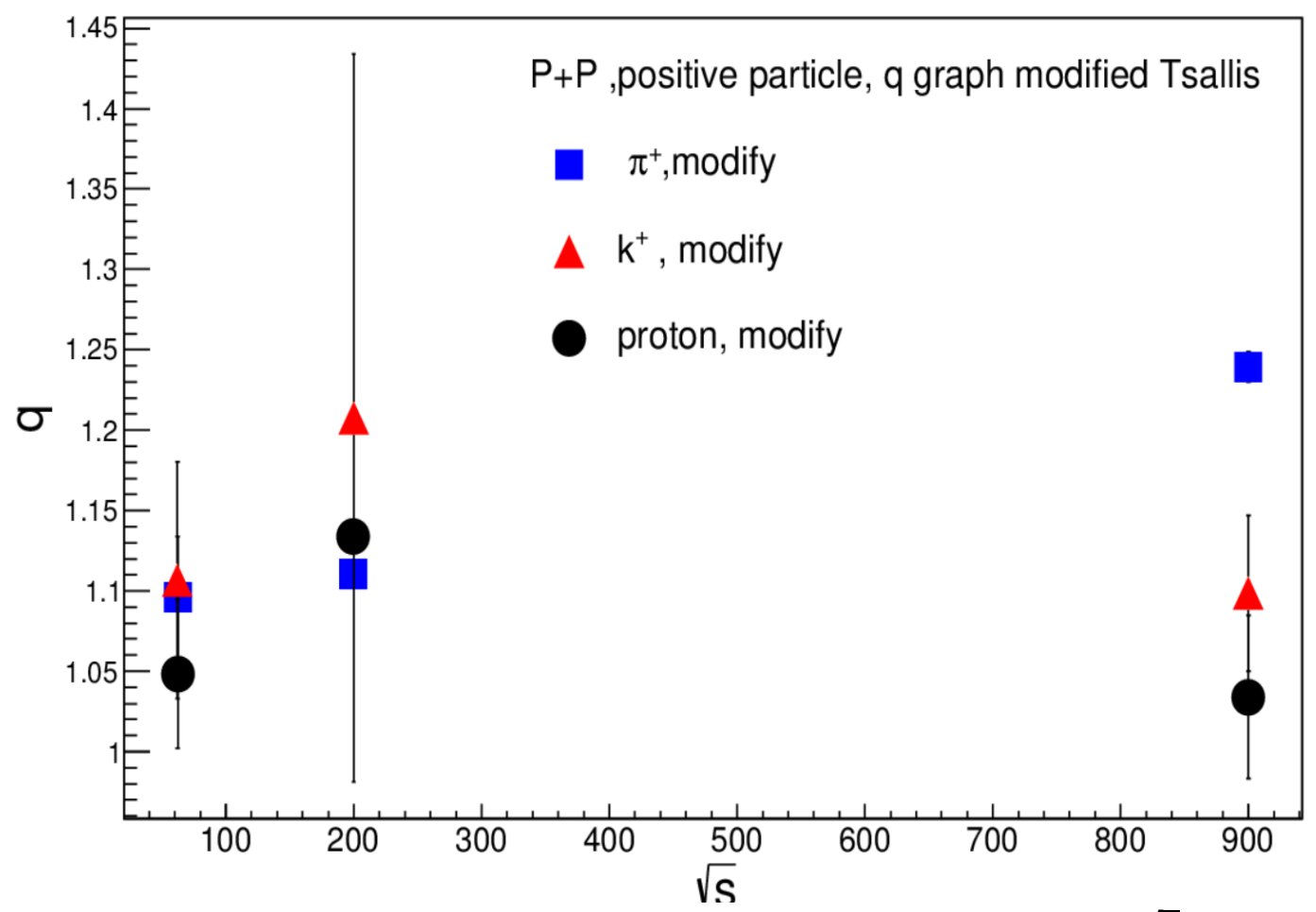

Figure 6: The nonextensive parameter $q$ for +ve hadron versus $\sqrt{s}$

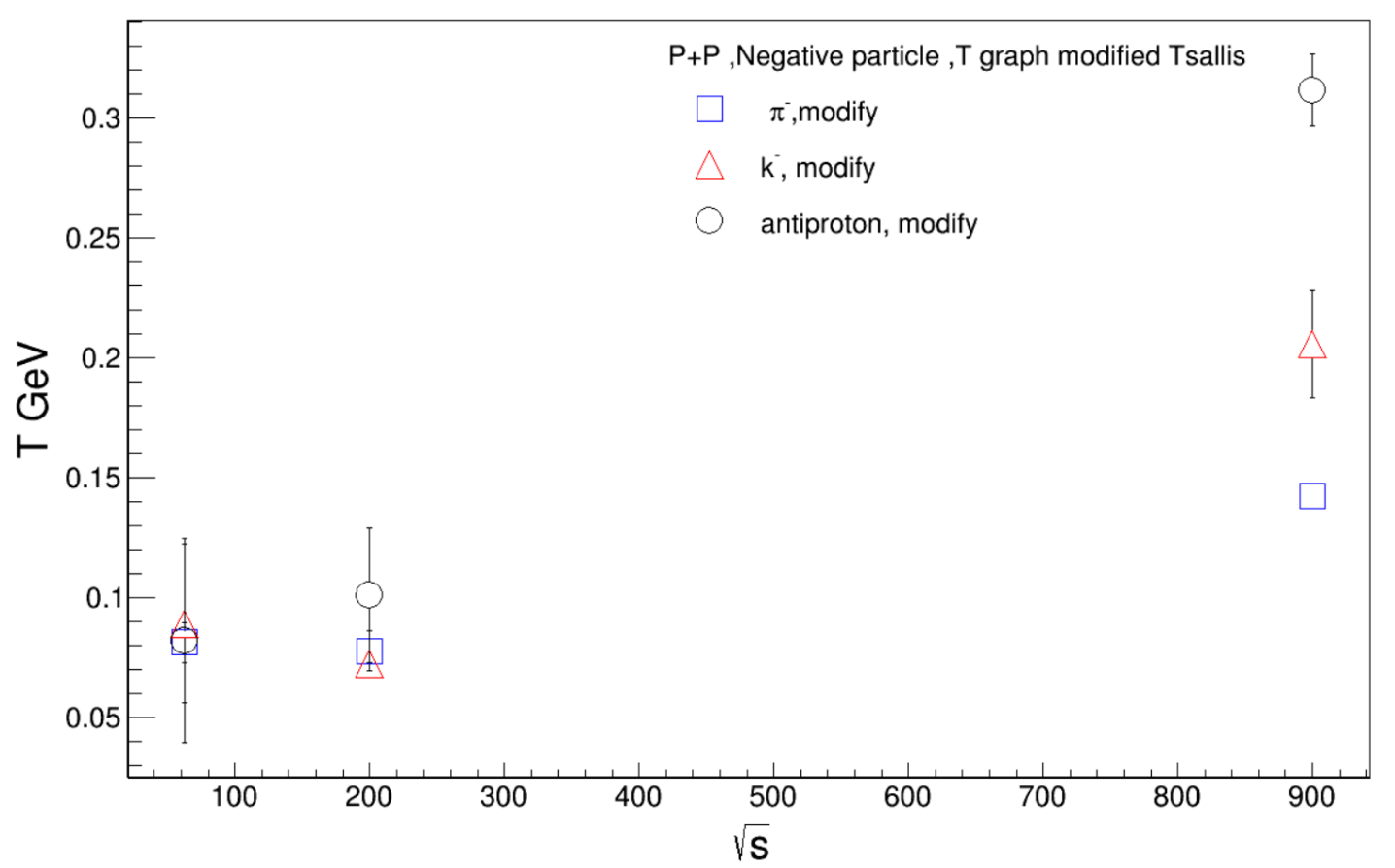

Figure 7: The Temperature parameter $T$ versus $\sqrt{s}$ 


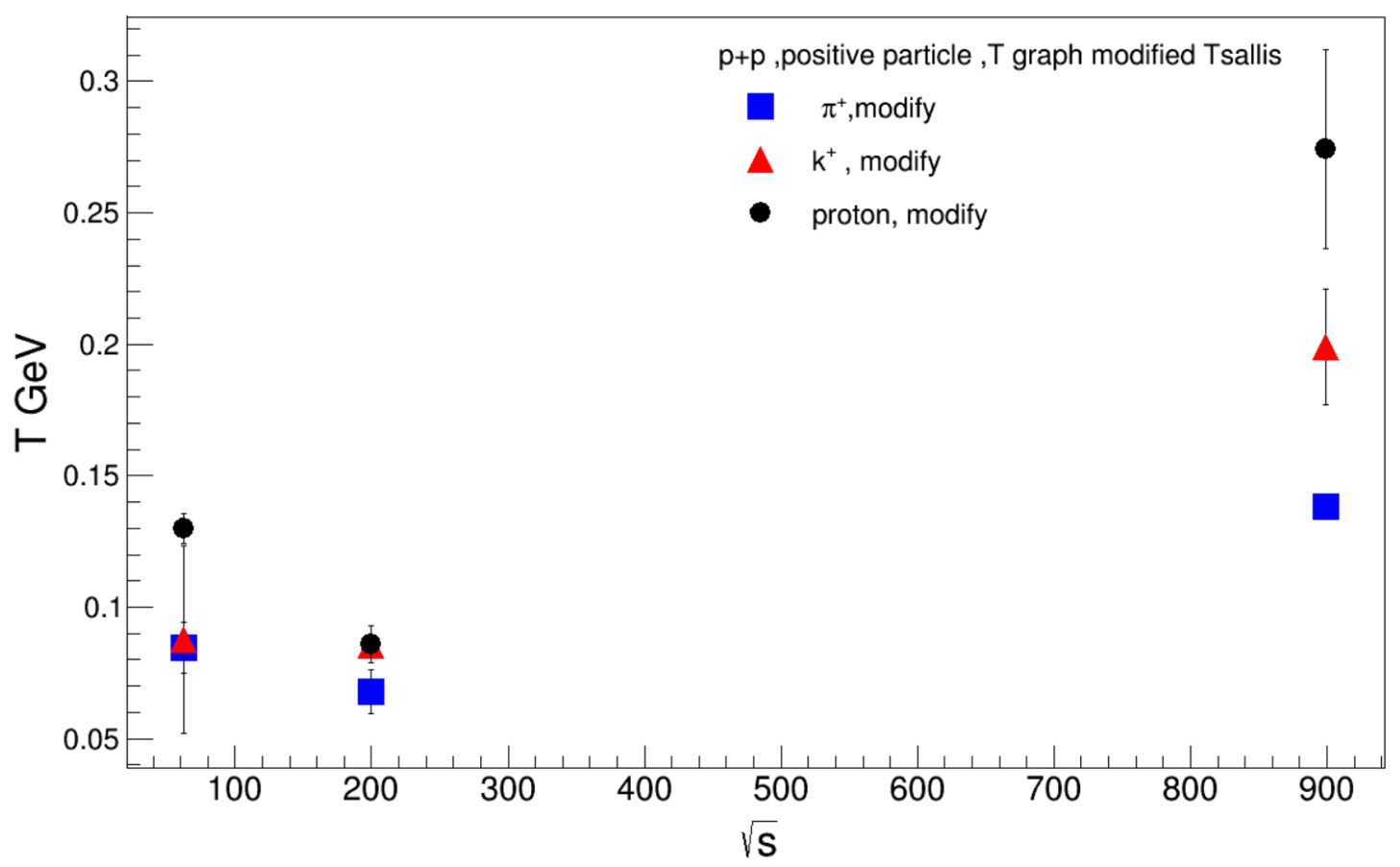

Figure 8: The temperature parameter $T$ versus $\sqrt{s}$

Now looking to the temperature parameters $T$ in tables $(1,2,3,4)$ and the corresponding figures $(9,10,14)$ we find that $T$ increases with particle mass such that $T_{\pi^{ \pm}}<T_{k^{ \pm}}<T_{p, \bar{p}}$. It is clearly indicates that the heavier particles have higher freeze-out temperatures as compared to lighter particles. Also the increases of temperature for $p / \bar{p}$ is more rapid as compared to measons $\left(\pi^{ \pm}, k^{ \pm}\right)$. The baryons in general freezeout earlier than mesons. This observation goes in-line with an intuitive expectation of mass dependent particle freeze-out or differential freeze-out. This is in agreement with the differential freeze-out scenarios proposed [D. Thaku et al ,2016]. Once all the unknown parameters inEq.(8) are given as we have shown and discussed. We can compute different observables.

In the current work we will limit ourselves to average transverse momentum $\left\langle p_{t}\right\rangle$ as a function of Hardon mass and energy. In order to do so we define the $k^{\text {th }}$ moment of the normalized distribution as

$$
\left\langle p_{t}^{k}\right\rangle=\frac{\int p_{t}^{k}\left(1+\frac{p_{t}}{\alpha T}\right)^{-\alpha} p_{t} d p_{t} d y d \emptyset}{\int\left(1+\frac{p_{t}}{\alpha T}\right)^{-\alpha} p_{t} d p_{t} d y d \emptyset}
$$

The $\varnothing$ dependence drops out since the distribution is symmetric with respect to $\varnothing$ as mention in section 1. 


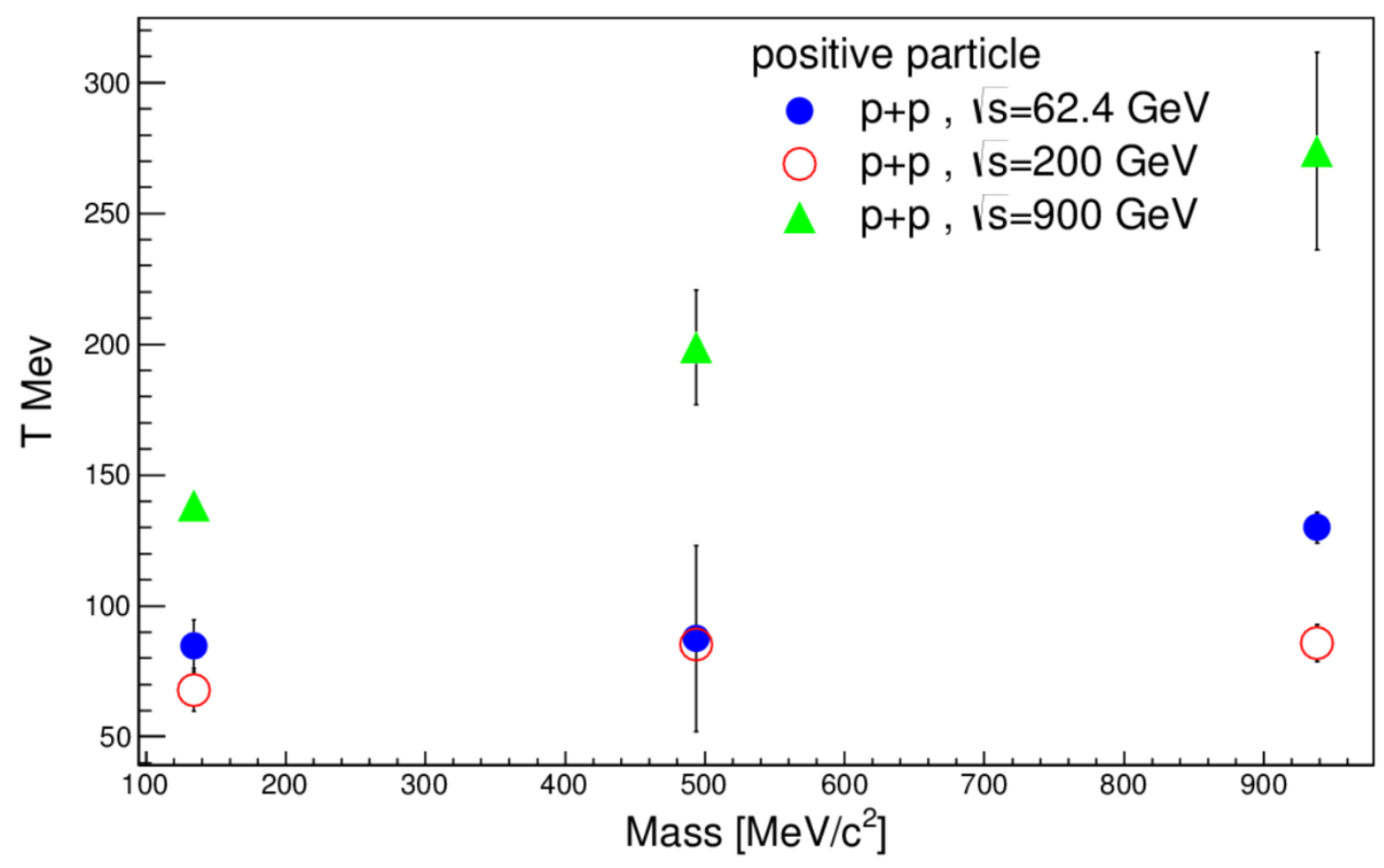

Figure 9: Temperature $T$ of positive hadrons versus their masses.

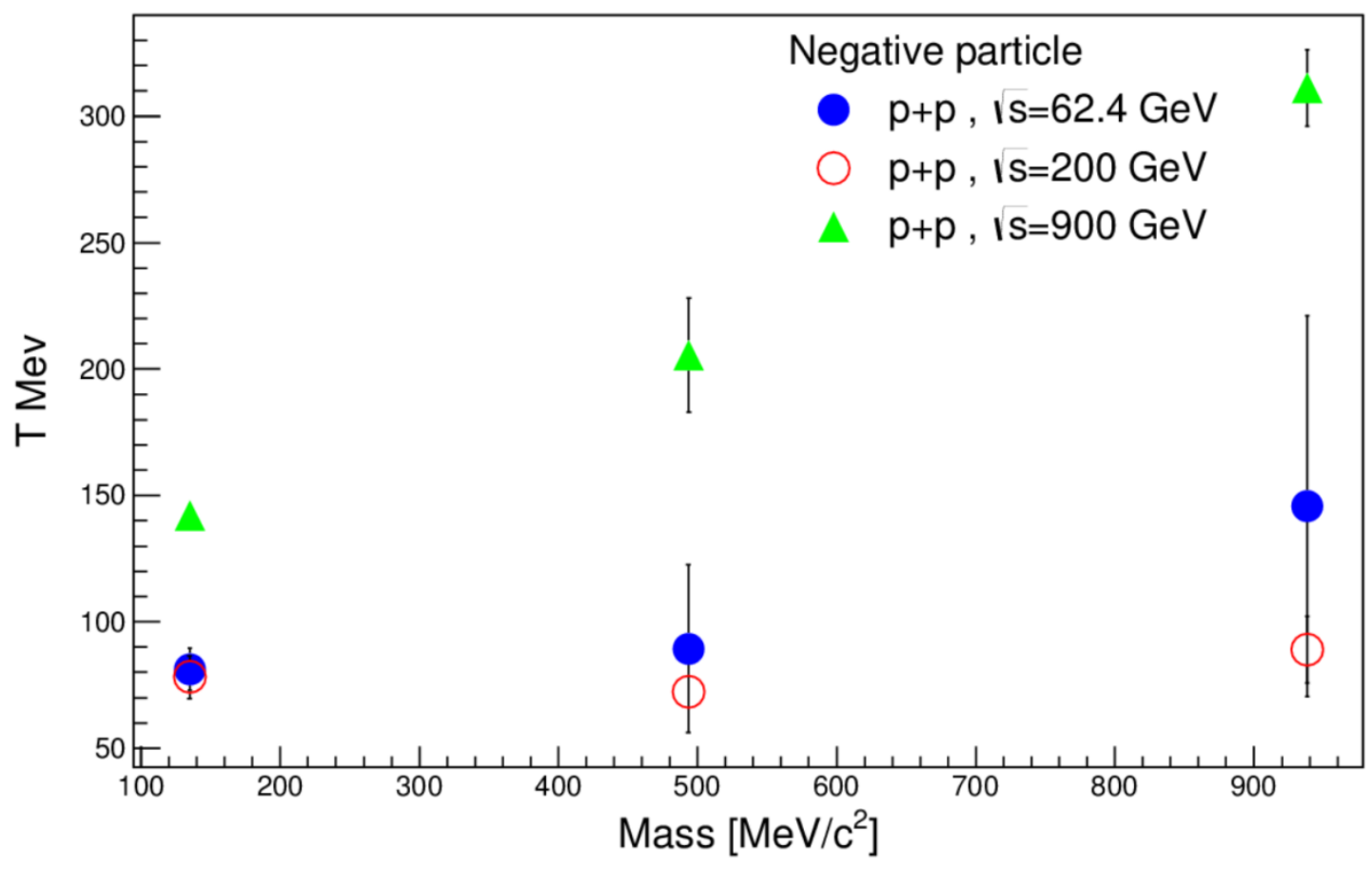

Figure 10: Temperature $T$ of negative hadrons versus their masses. 


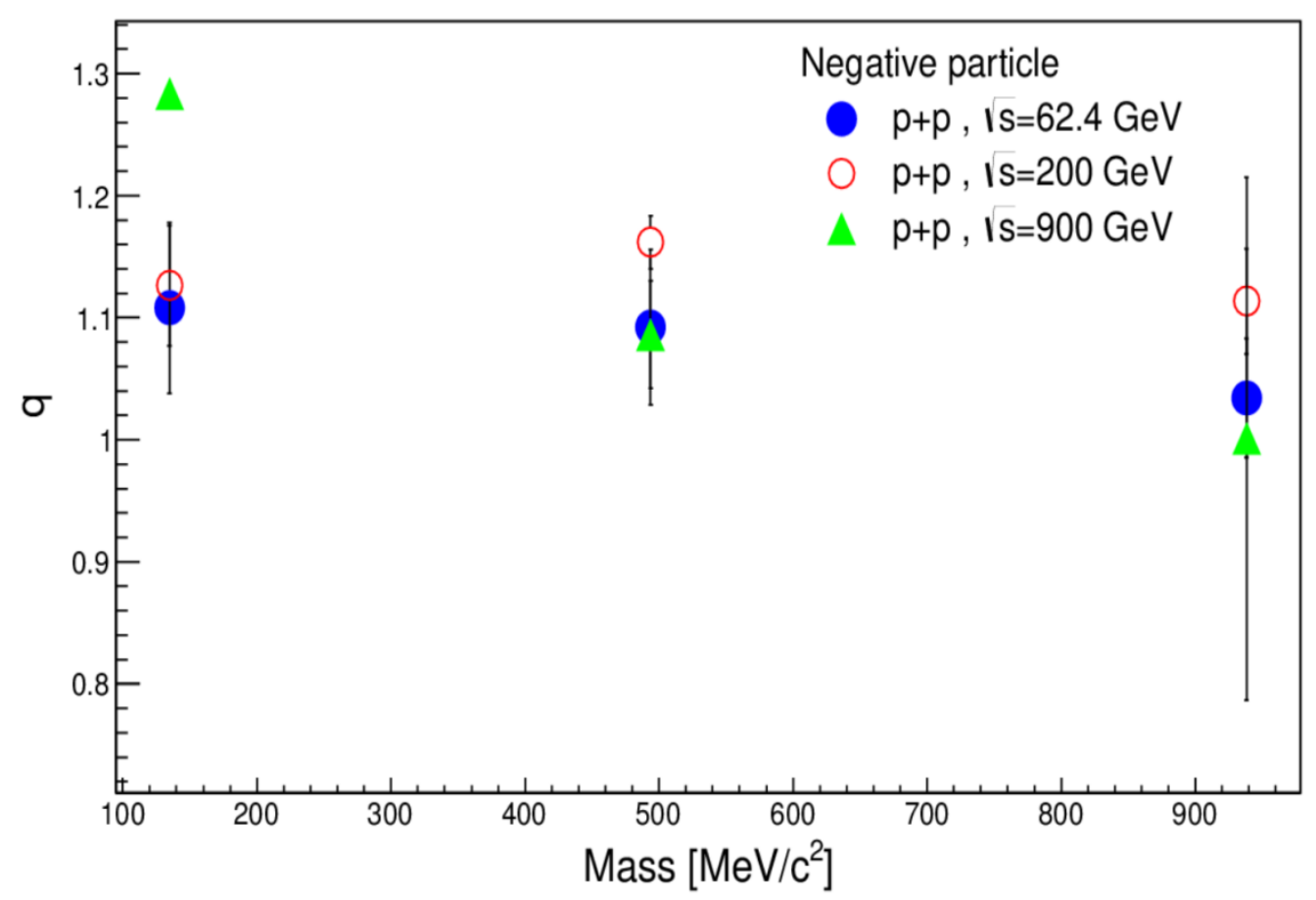

Figure 11: The nonextensive parameter $\mathrm{q}$ versus negative hadron mass

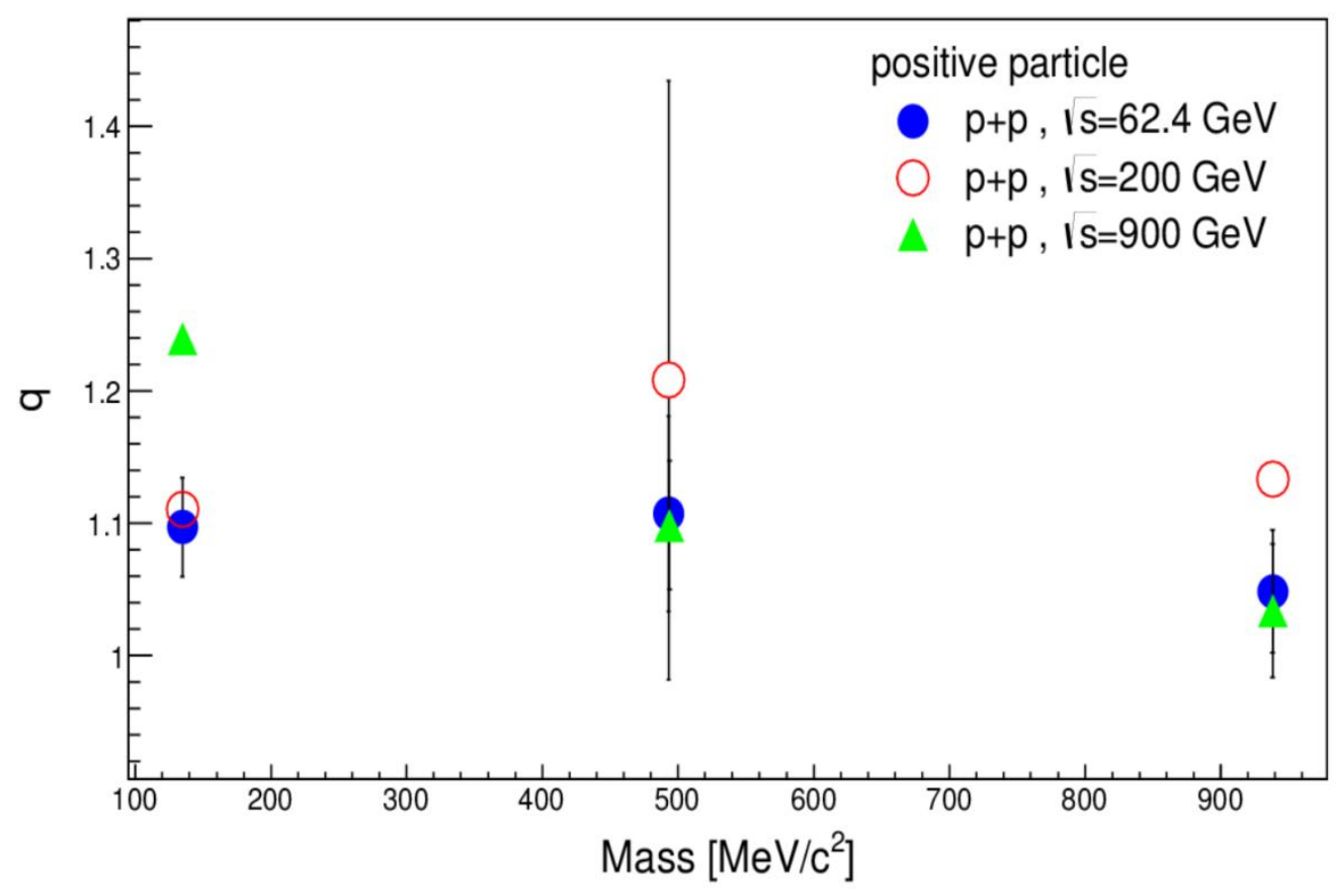

Figure 12: The nonextensive parameter q versus positive hadron mass 


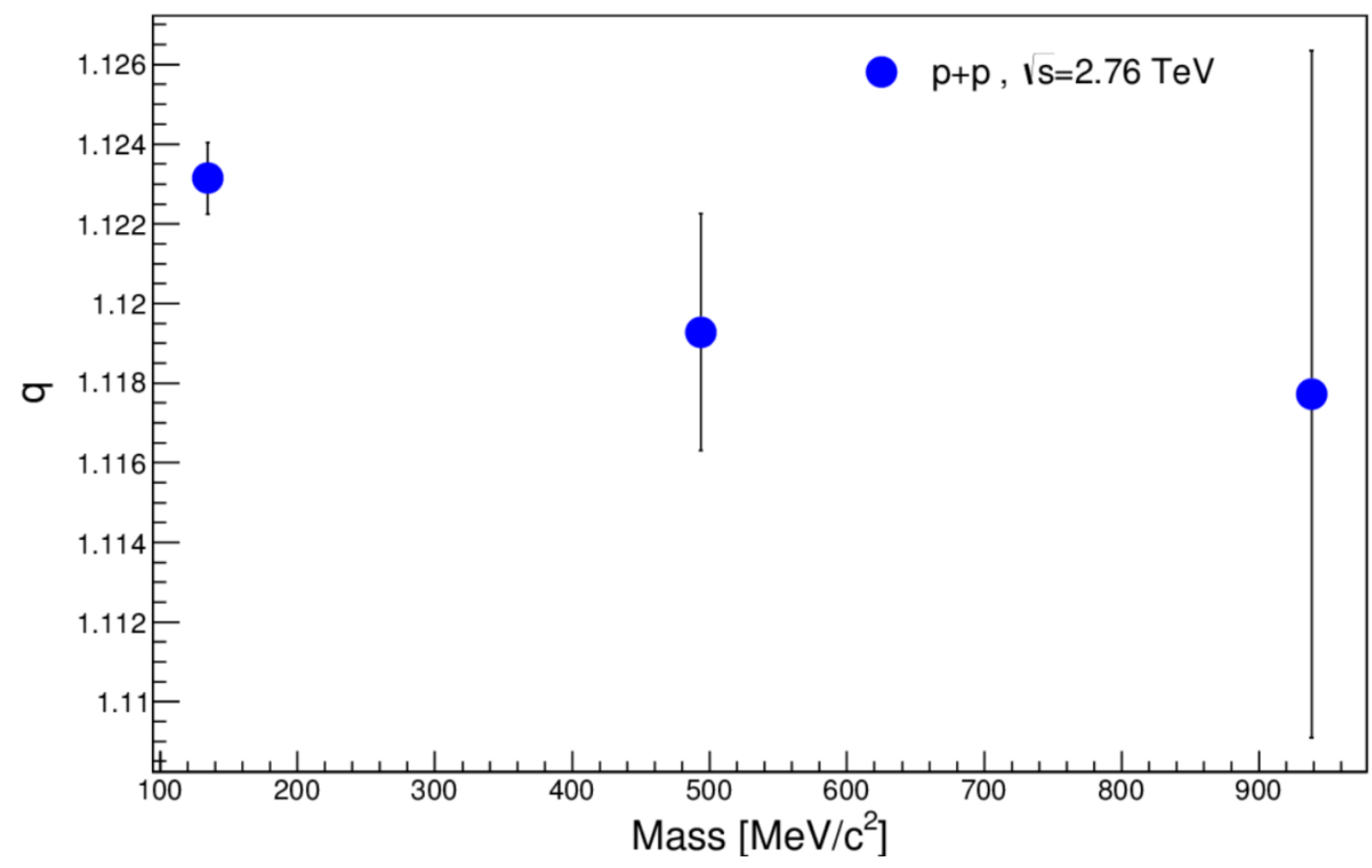

Figure 13: The nonextensive parameter q versus hadron mass

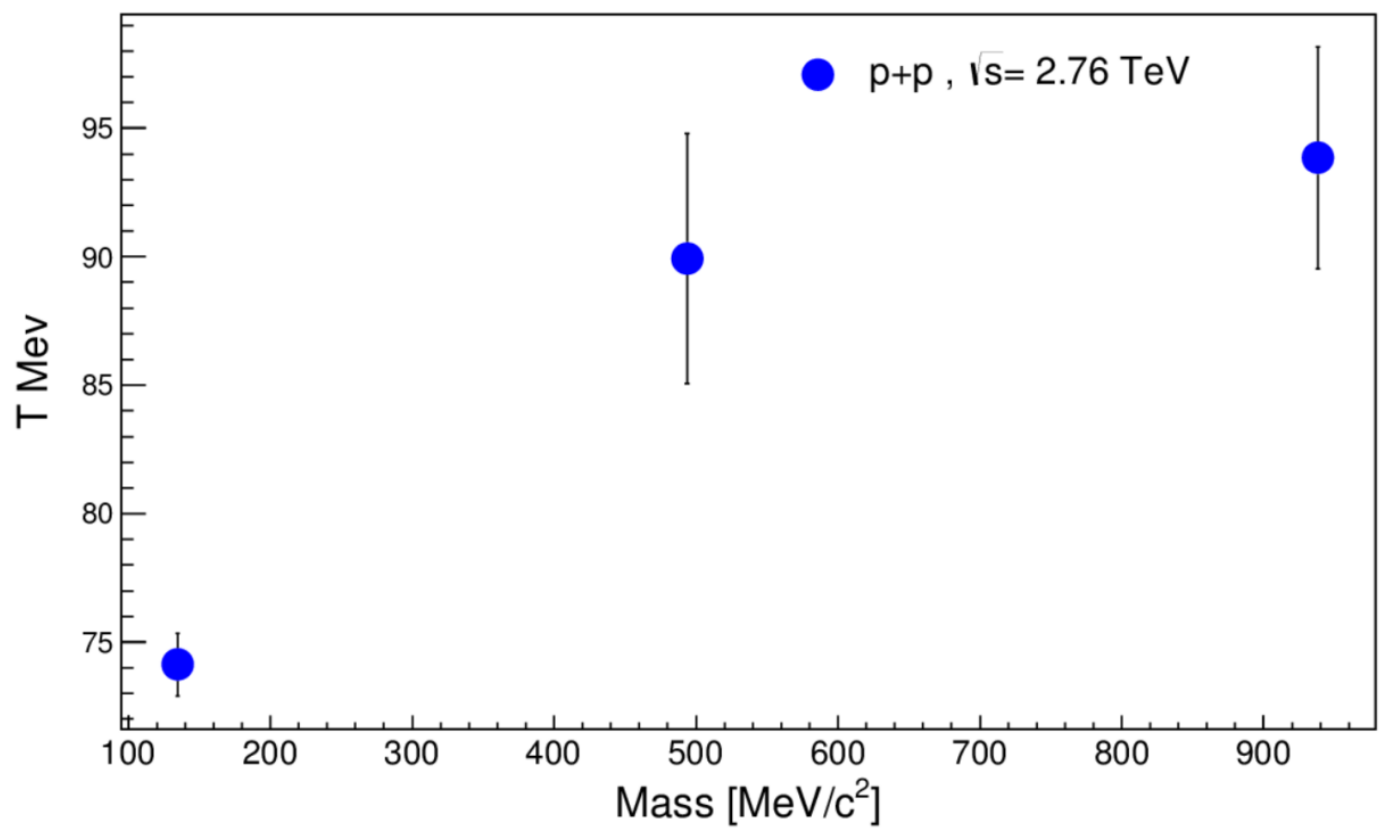

Figure 14: The temperature parameter $\mathrm{T}$ versus hadron mass. 


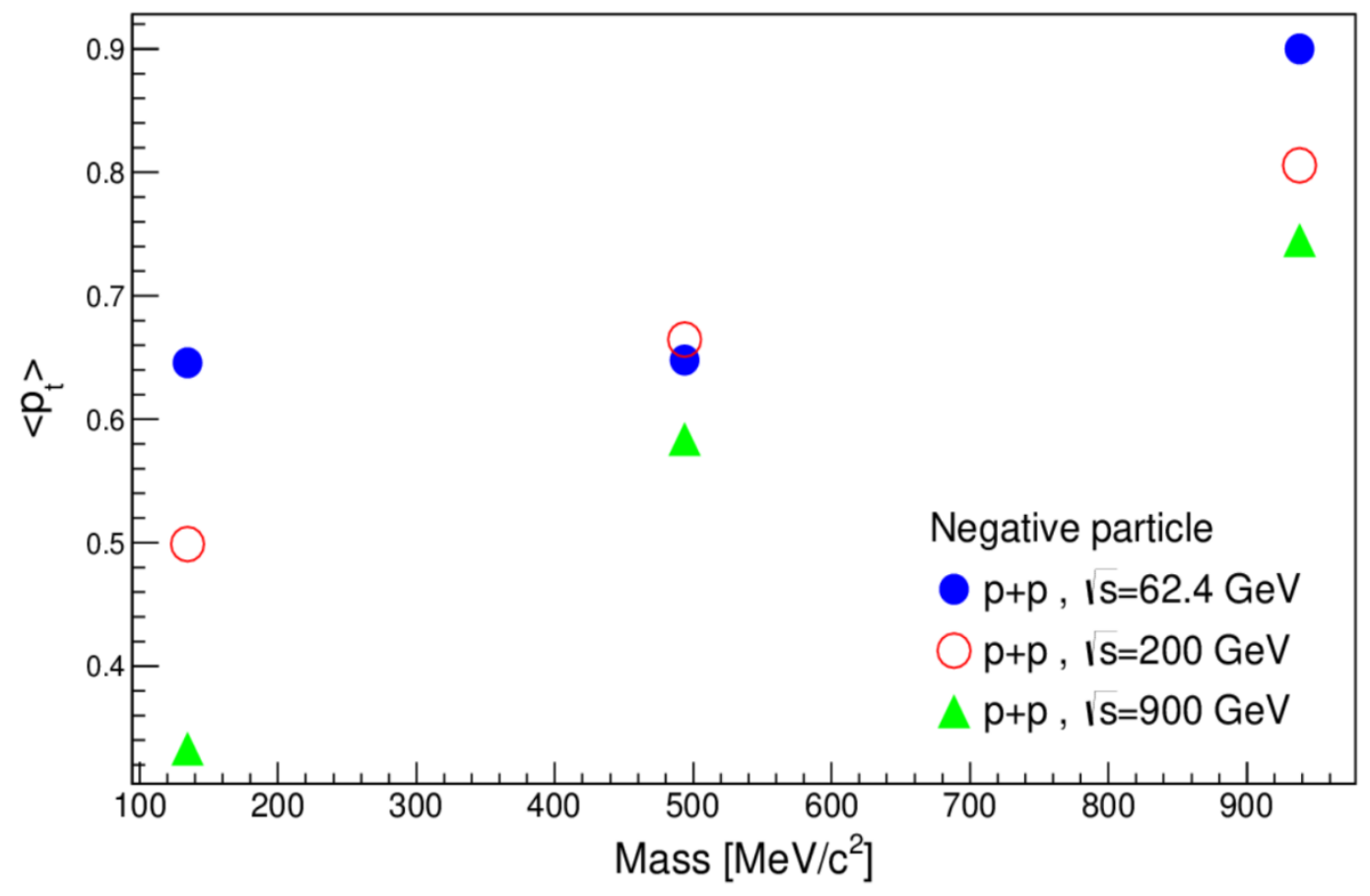

Figure 15: $\left\langle p_{t}\right\rangle$ of negative hadrons versus collisions energy

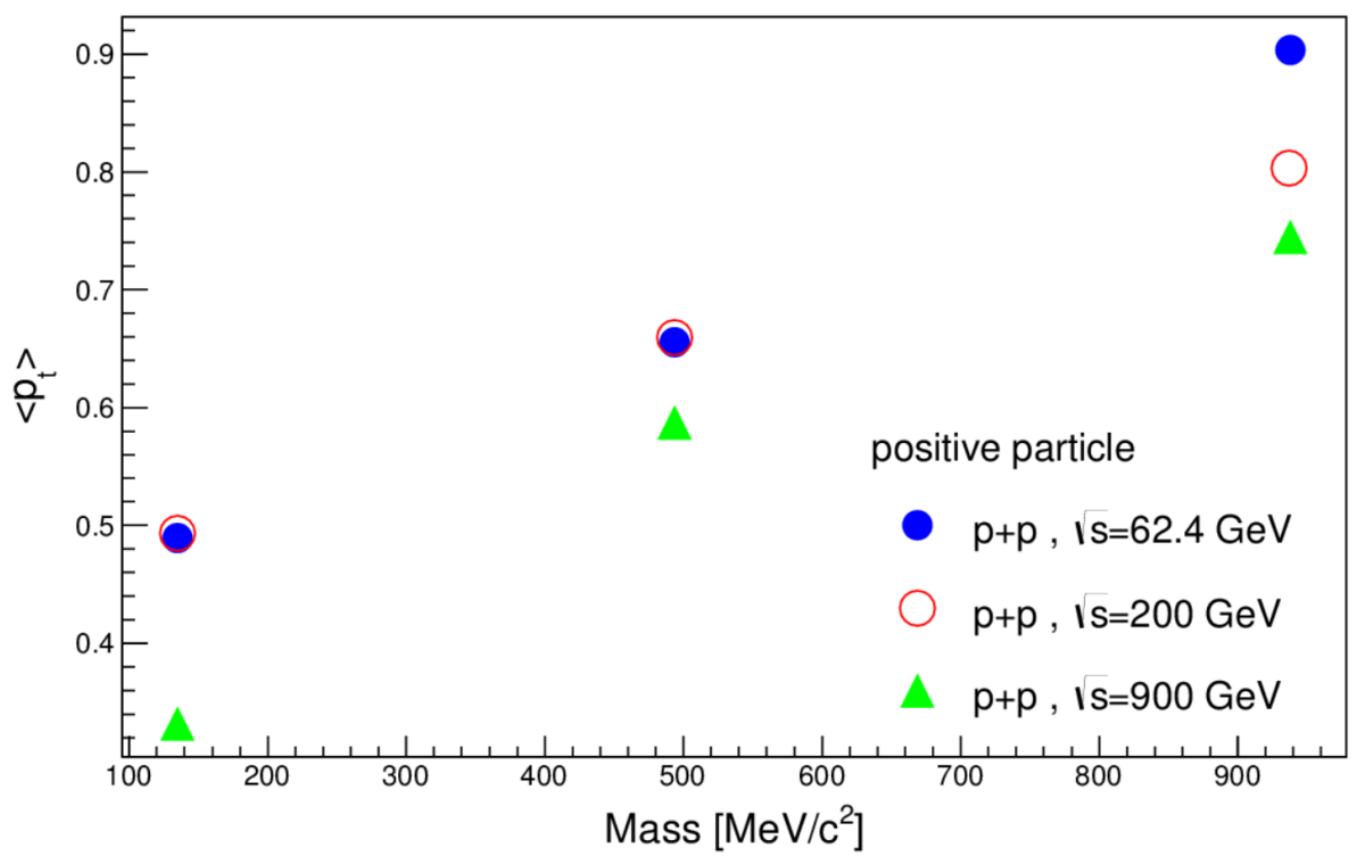

Figure 16: $\left\langle p_{t}\right\rangle$ of positive hadrons versus collisions energy 
The first moment $\mathrm{k}=1$ corresponds to $\left\langle\mathrm{p}_{\mathrm{t}}\right\rangle$ and the second moment $\mathrm{k}=2$ corresponds to $\left\langle p_{t}^{2}\right\rangle$ and the variance of the distribution $\operatorname{Var}\left(p_{t}\right)$ is given by

$$
\operatorname{Var}\left(p_{t}\right)=\left\langle p_{t}^{2}\right\rangle-\left\langle p_{t}\right\rangle^{2}
$$

In Fig.(15,16) we present the results of our calculations of $\left\langle p_{t}\right\rangle$ as a function of hadron mass using Eq.(10) with $\mathrm{k}=1$. We find that the $\left\langle\mathrm{p}_{\mathrm{t}}\right\rangle$ increases with increasing hadron mass such that $\left\langle\mathrm{p}_{\mathrm{t}}\right\rangle_{\pi^{ \pm}}\left\langle\left\langle\mathrm{p}_{\mathrm{t}}\right\rangle_{k^{ \pm}}\left\langle\left\langle\mathrm{p}_{\mathrm{t}}\right\rangle_{p, \bar{p}}\right.\right.$.

\section{Discussion and Conclusion}

In conclusion, the Pareto-Tsallis distribution, Eq.8 leads to an excellent description of data on transverse momentum distribution. Taken into account couple of approximations that we have taken such that we neglect the chemical potential of hadrons $\mu$ and both radial $\beta$ and elliptic flow velocity $v_{2}$. By comparing results from $62.4 \mathrm{Gev}$ at RHIC up to $2.7 \mathrm{TeV}$ at $\mathrm{LHC}$ it has been possible to extract hadron mass and energy dependence of the parameters $\mathrm{n}, \mathrm{q}$ and $\mathrm{T}$. We have used momentum dependent entropy index $\mathrm{n}$ that scales with $p_{t}$ dependent power ansatz. Parameters $\mathrm{T}$ and $\mathrm{q}$ were obtained by using the least square method. We investigated the collisions energy $\sqrt{s}$ dependence of the parameter values $q$ and $T$. The $\mathrm{q}$ parameters were found to be almost independent from $\sqrt{s}$ for protons and kaons and distributed around $q=1.1$ and $q$ is increasing for pions from 1.1 to almost 1.3 at top energy $\sqrt{s}=2.7 \mathrm{TeVUsing}$ the scaling TsallisPareto ansatz, the effective temperature, $T$ increases with collisionsenergy.

\begin{tabular}{|c|c|c|c|c|c|}
\hline \multicolumn{5}{|c|}{62.4 Gev PHENIX } \\
\hline particle & $\mathrm{T}$ & $\mathrm{q}$ & $\mathrm{K}$ & $\mathrm{A}$ & $\chi^{2} / n d f$ \\
\hline$\pi^{+}$ & $0.0846252 \pm 0.00986138$ & $1.09606 \pm 0.0375843$ & $0.0243774 \pm 0.562622$ & $1.00253 \pm 6.17546$ & 0.02524104 \\
\hline$\pi^{-}$ & $0.0812931 \pm 0.00832641$ & $1.10778 \pm 0.0699158$ & $0.00243036 \pm 0.237994$ & $1.00414 \pm 5.79771$ & 0.03234616 \\
\hline$k^{+}$ & $0.0875774 \pm 0.0355324$ & $1.10644 \pm 0.0736864$ & $0.0153147 \pm 0.637946$ & $1.03075 \pm 7.87473$ & 0.0345318666 \\
\hline$k^{-}$ & $0.0892701 \pm 0.0331675$ & $1.09250 \pm 0.0634979$ & $0.0537477 \pm 2.76598$ & $1.01748 \pm 8.96576$ & 0.0185472 \\
\hline $\mathrm{p}$ & $0.129970 \pm 0.00589840$ & $1.04847 \pm 0.0464307$ & $0.00492518 \pm 2.85060$ & $1.00008 \pm 6.41729$ & 0.0281819615 \\
\hline $\bar{p}$ & $0.145847 \pm 0.0753218$ & $1.03437 \pm 0.0488596$ & $0.0194563 \pm 2.88255$ & $1.22986 \pm 5.04656$ & 0.0433296153 \\
\hline
\end{tabular}

Table 1: $\chi^{2} / N D F$ and different extracted parameters of Eq.(8) for $\pi^{ \pm}, k^{ \pm}, p$ and $\bar{p}$ produced in ppcollisions as measured by PHENIX [A. Adare et al, 2011] $\sqrt{s_{N N}}=62.4 \mathrm{GeV}$.

\begin{tabular}{|c|c|c|c|c|c|}
\hline \multicolumn{5}{|c|}{ 200GeV PHENIX } \\
\hline particle & $\mathrm{T}$ & $\mathrm{q}$ & $\mathrm{K}$ & $\mathrm{A}$ & $\chi^{2} / n d f$ \\
\hline$\pi^{+}$ & $0.0678695 \pm 0.00817861$ & $1.11064 \pm 0.00697645$ & $3.36626 \pm 2.60700$ & $1.00009 \pm 8.62072$ & 0.0256206538 \\
\hline$\pi^{-}$ & $0.0779004 \pm 0.00843204$ & $1.12570 \pm 0.0493288$ & $0.0100805 \pm 0.160082$ & $1.00166 \pm 1.88810$ & 0.0285267307 \\
\hline$k^{+}$ & $0.0854086 \pm 0.00279072$ & $1.20751 \pm 0.226351$ & $0.360469 \pm 0.596220$ & $9.99999 \pm 6.40843$ & 0.0089581333 \\
\hline$k^{-}$ & $0.0722678 \pm 0.00285162$ & $1.16179 \pm 0.0215481$ & $0.00100298 \pm 0.154996$ & $1.00000 \pm 6.42031$ & 0.0322612666 \\
\hline $\mathrm{p}$ & $0.0858898 \pm 0.00698462$ & $1.13330 \pm 0.00964241$ & $0.156297 \pm 0.0206761$ & $9.96340 \pm 6.45932$ & 0.0533696969 \\
\hline $\bar{p}$ & $0.0890633 \pm 0.0131642$ & $1.11349 \pm 0.0432267$ & $0.0911787 \pm 0.264148$ & $6.51280 \pm 6.23151$ & 0.0331421212 \\
\hline
\end{tabular}

Table 2: $\chi^{2} / N D F$ and different extracted parameters of Eq.(8) for $\pi^{ \pm}, k^{ \pm}, p$ and $\bar{p}$ produced in ppcollisions as measured by PHENIX [A. Adare et al, 2011] $\sqrt{s_{N N}}=200 \mathrm{GeV}$. 


\begin{tabular}{|c|c|c|c|c|c|}
\hline \multicolumn{5}{|c|}{$900 \mathrm{Gev}$ LHC } \\
\hline particle & $\mathrm{T}$ & $\mathrm{q}$ & $\mathrm{K}$ & $\mathrm{A}$ & $\chi^{2} / n d f$ \\
\hline$\pi^{+}$ & $0.138230 \pm 0.00146616$ & $1.23934 \pm 0.00941634$ & $0.001 \pm 2.62331$ & $1.95096 \pm 0.131717$ & 0.2679753125 \\
\hline$\pi^{-}$ & $0.141800 \pm 0.00146408$ & $1.28270 \pm 0.00516747$ & $0.001 \pm 2.66860$ & $2.35196 \pm 0.141445$ & 0.2966096875 \\
\hline$k^{+}$ & $0.198814 \pm 0.0219545$ & $1.09856 \pm 0.0484162$ & $0.0544865 \pm 2.13119$ & $1.00114 \pm 6.59155$ & 0.1322776923 \\
\hline$k^{-}$ & $0.205488 \pm 0.0225403$ & $1.08618 \pm 0.0438780$ & $0.0474702 \pm 2.16696$ & $1.00000 \pm 6.39829$ & 0.1080511538 \\
\hline $\mathrm{P}$ & $0.273892 \pm 0.0377640$ & $1.03395 \pm 0.0505776$ & $0.131847 \pm 2.05474$ & $9.99930 \pm 6.36347$ & 0.0610578260 \\
\hline $\bar{p}$ & $0.311274 \pm 0.0150977$ & $1.00100 \pm 0.214201$ & $0.00100162 \pm 2.48665$ & $1.21121 \pm 6.98288$ & 0.0672960869 \\
\hline
\end{tabular}

Table 3: $\chi^{2} / N D F$ and different extracted parameters of Eq.(8) for $\pi^{ \pm}, k^{ \pm}, p$ and $\bar{p}$ produced in ppcollisions as measured by PHENIX [K. Aamodt et al, 2011] $\sqrt{s_{N N}}=900 \mathrm{GeV}$.

\begin{tabular}{|c|c|c|c|c|c|}
\hline \multicolumn{7}{|c|}{2.7 Tev LHC } \\
\hline particles & T & q & K & A & $\chi^{2} /$ NDF \\
\hline$\frac{\left\langle\pi^{+}+\pi^{-}\right\rangle}{2}$ & $0.740918 \pm 0.00122361$ & $1.12312 \pm 0.000904546$ & $3.49998 \pm 2.25268$ & $1.00000 \pm 0.0756364$ & 0.582008412 \\
\hline$\frac{\left\langle k^{-}+k^{+}\right\rangle}{2}$ & $0.899373 \pm 0.00486970$ & $1.11928 \pm 0.00297296$ & $3.41565 \pm 2.41247$ & $1.00349 \pm 7.71303$ & 0.025813859 \\
\hline$\frac{\langle p+\bar{p}\rangle}{2}$ & $0.936463 \pm 0.00431326$ & $1.11773 \pm 0.00862584$ & $0.00691535 \pm 0.00920205$ & $1.00000 \pm 0.587674$ & 0.074848958 \\
\hline
\end{tabular}

Table 4: Fit parameter for pp collisions at 2.7TeV LHC

Since increasing the collisions energy may result in a higher energy density and higher temperature. We estimated $T \approx 0.3 \mathrm{GeV}$ for $\mathrm{pp}$ collisions at $2.7 \mathrm{TeV} \mathrm{LHC}$ energy. further studies is needed to scan low energy pp collisions and get full picture on how the measured parameters depend on energy and hadronic masses and also take into account both radial and elliptic flow of identified hadrons.

\section{Reference}
A. Adare et al. [PHENIX Collaboration], Phys. Rev. C 83, 064903.
doi:10.1103/PhysRevC.83.064903 [arXiv:1102.0753 [nucl-ex]], (2011).
D. Thakur, S. Tripathy, P. Garg, R. Sahoo and J. Cleymans, Acta Phys. Polon. Supp. 9, 329. doi:10.5506/APhysPolBSupp.9.329 [arXiv:1603.04971 [hep-ph]], (2016).
D. Thakur, S. Tripathy, P. Garg, R. Sahoo and J. Cleymans, Adv. High Energy Phys. 2016, 4149352 .doi:10.1155/2016/4149352 [arXiv:1601.05223 [hep-ph]],(2016).

[A]G. G. Barnafoldi, K. Urmossy and T. S. Biro, J. Phys. Conf. Ser. 270, 012008. 
doi:10.1088/1742-6596/270/1/012008, (2011).

[B]G. G. Barnafoldi, T. S. Biro, K. Urmossy and G. Kalmar, Tsallis-Pareto-like distributions in hadron- hadron collisions, in Gribov-80,eds.Y.LDokshitzer,P.Levai and J.Nyiri . doi:10.1142/9789814350198-0034(world Scientific,2011).

G. Wilk and Z. Wlodarczyk, [arXiv:1701.06401 [hep-ph]],( 2017).

G. Wilk and Z. Wlodarczyk, Entropy 17, 384 .doi:10.3390/e17010384 [arXiv:1501.02608 [cond-mat.stat-mech]], (2015).

J. Cleymans, J. Phys. Conf. Ser. 779, no. 1, 012079 .doi:10.1088/1742-6596/779/1/012079 [arXiv:1609.02289 [hep-ph]], (2017).

K. Aamodt et al. [ALICE Collaboration], Eur. Phys. J. C 71, 1655 doi:10.1140/epjc/s10052011-1655-9 [arXiv:1101.4110 [hep-ex]] , (2011).

R. Hagedorn, Riv. NuovoCim. 6N10, 1. doi:10.1007/BF02740917, (1983).

R. J. Fries, S. A. Bass and B. Muller, Phys. Rev. Lett. 94, 122301

doi:10.1103/PhysRevLett.94.122301 [nucl-th/0407102], (2005). 


\title{
وصف تصادم بروتون-بروتون الغير قابل للاتساع عند الطاقات العالية
}

\author{
محمد حسن عبد العزيز 2,1 شيماء محمد عبد الر ازق3 منال سر اج3

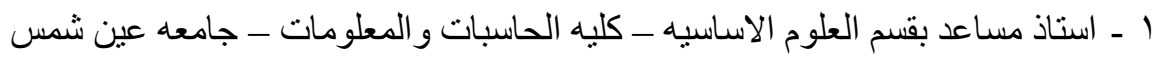

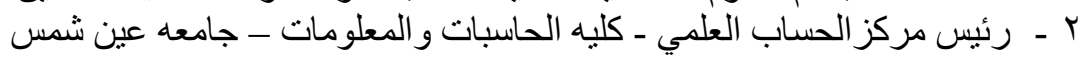

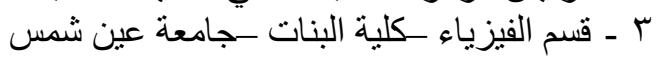

الملخص العربي

الاحصائيات الغير قابلة للأتساع تعطي تطبيق ممتاز في مجال تصادمات الطاقات العالية لدراسة الخو اص الحرارية للجسيمات الناتجة من التصادمات ـ في هذا البحث طبقنا معادلة (تساليس) المعايرة لدراسة معامل الانتروبي كدالة في كميه التحرك المستعرضه للهادرونات الناتجة. طبقنا توزيع تساليس-بارينو لدر اسة كميه التحرك المستعرضه

الهادرونات عi 2.7TeV توزيع تساليس -باريتوتقودنا الى وصف دقيق للبيانات في توزيع كميه التحرك المستعرضه. المعامل (q)

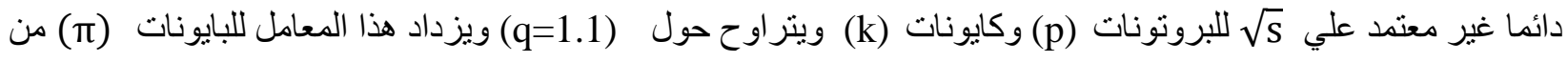
1.1 حو الي T = 0.3 Ge لتصادم بروتون-بروتون عند طاقة الهصادم الهييدروني الكبير 2.7 TeV الهادرونات المختلفة لها درجات حرارة مؤثرة مختلفة مثلا الهادرونات الثقبلة لها اعلي درجة حرارة تجمد خارجي . 